\title{
The effect of systemic treatments on periostin expression reflects their interference with the eosinophilic inflammation in chronic rhinosinusitis with nasal polyps*
}

\author{
Els De Schryver', Lara Derycke', Lien Calus'1, Gabriele Holtappels', \\ Peter W. Hellings ${ }^{1,3,4}$, Thibaut Van Zele', Claus Bachert ${ }^{1,2}$, Philippe Gevaert ${ }^{1}$ \\ ' Upper Airways Research Laboratory, Department Otorhinolaryngology, Ghent University Hospital, Ghent, Belgium \\ 2 Division of ENT Diseases, Clintec, Karolinska Institutet, Stockholm, Sweden \\ ${ }^{3}$ Clinical Division of ENT, Head and Neck Surgery, Laboratory of Clinical Immunology, University Hospitals Leuven, Belgium \\ ${ }^{4}$ Department of Otorhinolaryngology, Academic Medical Center, Amsterdam, the Netherlands
}

Rhinology 55: 152-160, 2017

https://doi.org/10.4193/Rhino16.314

*Received for publication:

October 31, 2016

Accepted: January 19, 2017

\begin{abstract}
Background: Periostin is a recently discovered biomarker for eosinophilic inflammation. Chronic rhinosinusitis with nasal polyps is a T-helper 2-skewed chronic inflammatory airway disease. Medical treatments aim to relieve symptoms and maintain clinical control by interfering with the inflammatory cascade. The effect on nasal and serum periostin levels is however yet unknown. We aimed to evaluate the effect of omalizumab, mepolizumab, methylprednisolone and doxycycline on nasal and systemic periostin expression.
\end{abstract}

Methods: This study is based on 3 previously published trials. Nasal and systemic periostin were assessed in CRSwNP patients, randomly assigned to receive doxycycline $(n=14)$, methylprednisolone $(n=14)$, mepolizumab $(n=20)$ or omalizumab $(n=15)$. There was a control group for each treatment scheme. Doxycycline (200 mg on the first day, followed by $100 \mathrm{mg}$ once daily) and methylprednisolone (32-8 mg once daily) were administered during 20 days; mepolizumab was injected at baseline and at 4 weeks. Omalizumab was injected every 2 or 4 weeks, following the official drug leaflet.

Results: Methylprednisolone and omalizumab significantly reduced serum periostin levels at 4 and 8 weeks, respectively, after the start of the treatment. The effect of methylprednisolone was transient. Nasal periostin levels decreased significantly after 8 weeks of treatment with mepolizumab. The periostin expression is in accordance with the previously reported effect on the eosinophilic inflammation and clinical outcome.

Conclusion: All treatment options distinctly influence periostin expression, reflecting the interference with the local or systemic eosinophilic inflammatory cascade.

Key words: chronic rhinosinusitis with nasal polyps, doxycycline, methylprednisolone, monoclonal antibodies, periostin

\section{Introduction}

Chronic rhinosinusitis with nasal polyps (CRSwNP) is a chronic inflammatory disease affecting $4 \%$ of the general population ${ }^{(1)}$ with an overall recurrence rate of $60 \%{ }^{(2)}$. Although the inflammatory pattern varies and different endotypes exist, a T helper
2 (TH2) inflammation with eosinophilia, local IgE upregulation and oedematous tissue remodelling are striking features in most CRSwNP patients ${ }^{(3,4)}$. Interleukin (IL)-5 and eosinophilic cationic protein (ECP), seem to be crucial in the regulation of the eosinophilic inflammation. The sIL-5Ra receptor mediates the biolo-

Abbreviations: CFB: change from baseline; CRSwNP: chronic rhinosinusitis with nasal polyps; ECP: eosinophilic cationic protein; FLC: free light chains; IFN- : interferon-gamma; IgE: Immunoglobulin E; IL-5: interleukin-5; MMP-9: matrix metalloproteinase-9; MPO: myeloperoxidase; NERD: NSAIDexacerbated Respiratory Disease; sIL-5Ra: soluble interleukin-5 receptor alpha; TH1: T helper 1; TH2: T helper 2; TPS: total polyp score; TSS: total symptom score 
gical signal of IL-5 and this receptor is more abundant in nasal polyps as compared to control tissue ${ }^{(5)}$. MMPs play an important role during inflammation by degrading extracellular matrix components. MMP-9 is elevated and has a known pathogenic effect in CRSwNP ${ }^{(6-8)}$. Periostin is a quite recently discovered marker downstream of IL-4-/IL-13-signals. It is considered a biomarker for eosinophilic inflammation in airway disease ${ }^{(9,10)}$ that could help to refine CRSwNP phenotypes. A serum periostin cut-off value of $25 \mathrm{ng} / \mathrm{mL}$ was suggested to predict airway eosinophilia in asthmatics ${ }^{(10)}$. Furthermore, the EXTRA study reported $50 \mathrm{ng} /$ $\mathrm{mL}$ as the median serum periostin value in 534 patients with uncontrolled severe persistent asthma ${ }^{(11)}$.

Understanding of the pathogenesis of nasal polyps has increased tremendously over the past decades, but the management of nasal polyps remains challenging. Non-surgical treatment is a life long necessity to control symptoms, infections and recurrences. When a combination of saline irrigation, topical nasal steroids/oral steroids, and an $\neg$ tibiotics is not sufficient surgery is indicated. Even after surgical removal of the polyps recurrences are common ${ }^{(2)}$. For patients necessitating repeated surgery $\mathrm{TH}_{2}-$ directed treatments, including omalizumab and mepolizumab become of interest. Though, these treatments are only effective in a selected population.

Glucocorticosteroids have potent anti-inflammatory effects. There is strong evidence for intranasal steroids in the treatment of nasal polyps, which is the standard first-line treatment ${ }^{(12)}$. In case of insufficient disease control, oral corticosteroid courses are prescribed. It is generally accepted that methylprednisolone has an important effect on nasal symptoms and polyp size ${ }^{(13)}$. Secondly, doxycycline is commonly prescribed to treat nasal polyps. The rationale is that doxycycline exerts biological functions independent from their antimicrobial effect. Importantly, doxycycline is suitable to treat Staphylococcus aureus ${ }^{(14)}$. A chronic colonization with this microorganism is much more frequent in patients with nasal polyps than in the general population and this is thought to contribute to maintenance of inflammation and disease progression.

Inhibition of IL-5 with for example mepolizumab, a humanized anti-IL-5 monoclonal antibody, is a potential therapeutic approach in patients with severe eosinophilic nasal polyposis ${ }^{(15)}$. Indeed, IL-5 is the most important eosinophilopoietin, with a role in the production, recruitment, activation and survival of eosinophils.

Finally, blocking lgE, with for example omalizumab, a human anti-IgE monoclonal antibody is a novel medical treatment option for certain patients with nasal polyps ${ }^{(16)}$. Local IgE formation has been described in nasal polyps. This IgE is functional and also thought to play a key role in pathogenesis. Omalizumab can be indicated to treat adults and children 12 years of age and older with moderate to severe persistent asthma or chronic idiopathic urticaria if symptoms are not controlled by conventi- onal treatment.

We previously demonstrated the effectiveness of these treatments in CRSwNP by means of randomized placebo-controlled studies $^{(14,15,17)}$. Here we assess the influence of these drugs on serum periostin. Indeed, serum periostin levels are shown to decrease in asthma after treatment with oral prednisolone ${ }^{(18)}$ and lebrikizumab, an anti-IL-13 monoclonal antibody ${ }^{(19)}$. Furthermore, this marker could hold promise in predicting responsiveness to TH2-directed therapies, as suggested for asthma. For example, high levels of periostin predict response to omalizumab ${ }^{(11)}$ and to lebrikizumab ${ }^{(20,21)}$ in severe refractory asthma. We aimed to evaluate the effect of omalizumab, mepolizumab, methylprednisolone and doxycycline on nasal and systemic periostin expression, as this could provide additional information on the mode of action.

\section{Materials and methods}

\section{Study design}

This study is based on three previously published double-blind randomised controlled trials (RCTs). In these RCTs patients were randomly assigned to receive either: methylprednisolone capsules, doxycycline capsules, intravenous injections of mepolizumab, subcutaneous injections of omalizumab, or placebo ${ }^{\text {(14-16). }}$ A corresponding placebo control group was provided for each dosage scheme of the interventional medication in each study separately (Figure E1). In total, 100 patients with bilateral nasal polyps were included. Doxycycline (200 mg on the first day, followed by $100 \mathrm{mg}$ once daily) and methylprednisolone (32-8 mg once daily) were administered during 20 days; mepolizumab was injected at baseline and at 4 weeks. Omalizumab was injected every 2 or 4 weeks, following the official drug leaflet. Full details on the trials are described in the online repository.

The effect of these treatments on periostin, the inflammatory cascade and clinical outcome were put in respect to each other

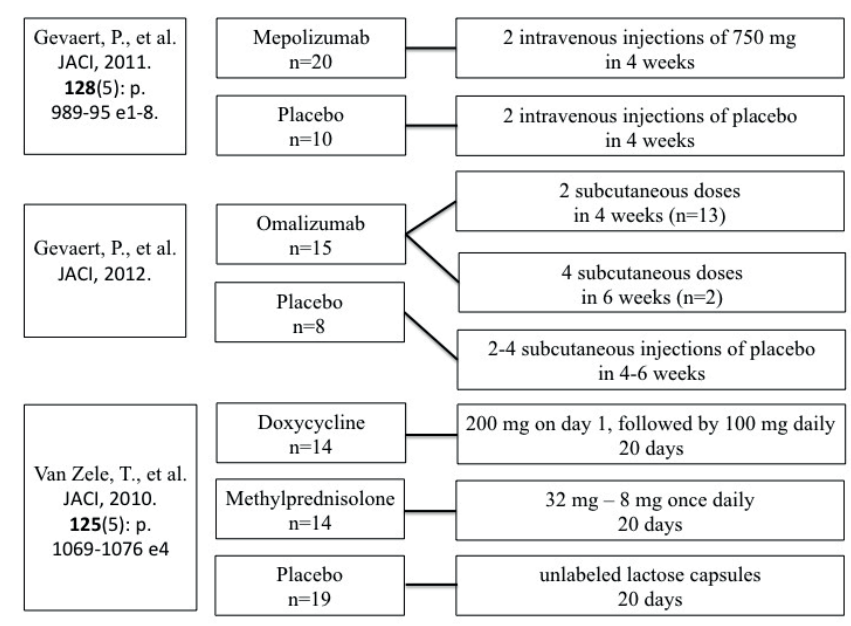

Figure E1. Study design. 


\begin{tabular}{|c|c|c|c|c|c|}
\hline & PLACEBO & OMALIZUMAB & MEPOLIZUMAB & $\begin{array}{c}\text { METHYL- } \\
\text { PREDNISOLONE }\end{array}$ & DOXYCYCLINE \\
\hline \multicolumn{6}{|c|}{ GENERAL CHARACTERISTICS } \\
\hline Number, $\mathrm{n}$ & 37 & 15 & 20 & 14 & 14 \\
\hline Gender, Female/male & $10 / 27$ & $3 / 12$ & $6 / 14$ & $2 / 12$ & $3 / 11$ \\
\hline Age, Mean (SD) & $50(12.48)$ & 49 (9.69) & $50(8.86)$ & 49 (12.08) & $52(17.68)$ \\
\hline Allergy, n (\%) & $20(54.1)$ & $6(40)$ & $10(50)$ & $6(42.9)$ & $2(14.3)$ \\
\hline Asthma, n(\%) & $16(43.2)$ & $15(100)$ & $10(50)$ & $6(42.9)$ & $4(28.6)$ \\
\hline NERD, n(\%) & $9(24.3)$ & $8(53.3)$ & $5(25.0)$ & $2(14.3)$ & $1(7.1)$ \\
\hline \multicolumn{6}{|c|}{ CLINICAL CHARACTERISTICS } \\
\hline TSS Median(IQR) & $8.5(6.0-9.0)$ & $7(6.0-10.0)$ & $8(7.0-9.0)$ & $7(4.0-8.3)$ & $5.5(4.0-10.0)$ \\
\hline TPS Median(IQR) & $6(5.3-7.0)$ & $6(4.0-6.0)$ & $6(4.0-6.0)$ & $6(5.8-6.0)$ & $6(4.8-7.0)$ \\
\hline Previous FESS n(\%) & $24(64.9)$ & $13(86.7)$ & $16(80.0)$ & $11(78.6)$ & $6(42.9)$ \\
\hline Recurrence, n (\%) & $23(62.2)$ & $13(86.7)$ & $14(70.0)$ & $11(78.6)$ & $6(42.9)$ \\
\hline Smoking, n (\%) & $4(11.1)$ & $2(13.3)$ & $5(25.0)$ & $4(28.6)$ & $3(21.4)$ \\
\hline \multicolumn{6}{|c|}{ NASAL SECRETIONS } \\
\hline $\begin{array}{l}\text { Periostin }(\mathrm{ng} / \mathrm{mL}) \\
\text { Mean (SEM) }\end{array}$ & 1099.39 (239.77) & $1253.78(504.63)$ & $818.63(331.73)$ & $617.43(241.27)$ & 455.75 (241.69) \\
\hline $\begin{array}{l}\text { Total lgE (kU/L) } \\
\text { Mean (SEM) }\end{array}$ & $140.88(50.54)$ & $49.85(16.62)$ & $63.52(28.58)$ & $84.76(43.52)$ & $23.84(11.76)$ \\
\hline $\begin{array}{l}\text { IL-5 (pg/mL) } \\
\text { Mean (SEM) }\end{array}$ & $100.32(23.83)$ & $90.43(27.14)$ & $96.94(23.75)$ & $46.49(20.78)$ & $38.06(27.71)$ \\
\hline $\begin{array}{l}\mathrm{ECP}(\mu \mathrm{g} / \mathrm{L}) \\
\text { Mean }(\mathrm{SD})\end{array}$ & $601.54(113.57)$ & $452.56(94.35)$ & 914.57 (199.37) & $515.62(229.63)$ & $853.57(600.52)$ \\
\hline $\begin{array}{l}\text { slL-5Ra }(p g / m L) \\
\text { Mean (SEM) }\end{array}$ & $771.10(160.03)$ & $664.30(188.55)$ & $586.28(178.94)$ & $318.32(82.66)$ & $426.01(125.23)$ \\
\hline $\begin{array}{l}\text { MPO (ng/mL) } \\
\text { Mean (SEM) }\end{array}$ & $6036.57(1953.14)$ & $3585.78(1205.07)$ & $10612.83(3944.80)$ & 18539.35 (11848.27) & $22001.32(14589.26)$ \\
\hline $\begin{array}{l}\text { MMP9 }(\mathrm{ng} / \mathrm{mL}) \\
\text { Mean (SEM) }\end{array}$ & $4990.24(1432.44)$ & $5504.12(1477.73)$ & $8464.32(2852.27)$ & $5440.04(2811.37)$ & $9398.88(6283.58)$ \\
\hline $\mathrm{SAE} \lg \mathrm{E}, \mathrm{n}(\%)$ & $3(8.6)$ & $1(6.7)$ & $1(5.6)$ & $2(14.3)$ & $1(7.1)$ \\
\hline \multicolumn{6}{|c|}{ SERUM } \\
\hline $\begin{array}{l}\text { Periostin }(\mathrm{ng} / \mathrm{mL}) \\
\text { Mean (SEM) }\end{array}$ & $81.08(3.77)$ & $90.03(12.45)$ & $79.15(6.15)$ & $73.86(5.13)$ & 69.91 (3.89) \\
\hline $\begin{array}{l}\text { Total lgE (kU/L) } \\
\text { Mean (SEM) }\end{array}$ & $169.78(44.59)$ & $129.08(25.91)$ & $204.45(79.53)$ & $308.81(82.90)$ & $78.55(21.71)$ \\
\hline $\begin{array}{l}\text { ECP }(\mu \mathrm{g} / \mathrm{L}) \\
\text { Mean (SEM) }\end{array}$ & $34.00(5.28)$ & $37.27(7.62)$ & $40.64(6.30)$ & $19.78(3.18)$ & $14.37(2.05)$ \\
\hline $\begin{array}{l}\text { sIL-5Ra }(\mathrm{pg} / \mathrm{mL}) \\
\text { Mean (SEM) }\end{array}$ & $289.38(21.73)$ & $324.23(58.35)$ & $348.42(28.85)$ & $303.41(21.83)$ & $267.50(30.73)$ \\
\hline $\begin{array}{l}\text { Eosinophils/ } \mu \mathrm{L} \\
\text { Mean (SEM) }\end{array}$ & $433.00(42.68)$ & $519.93(74.80)$ & $408.45(53.00)$ & $476.43(72.06)$ & $320.57(59.71)$ \\
\hline SAE IgEn (\%) & $20(55.6)$ & $9(60.0)$ & $8(40.0)$ & $11(78.6)$ & $5(35.7)$ \\
\hline
\end{tabular}



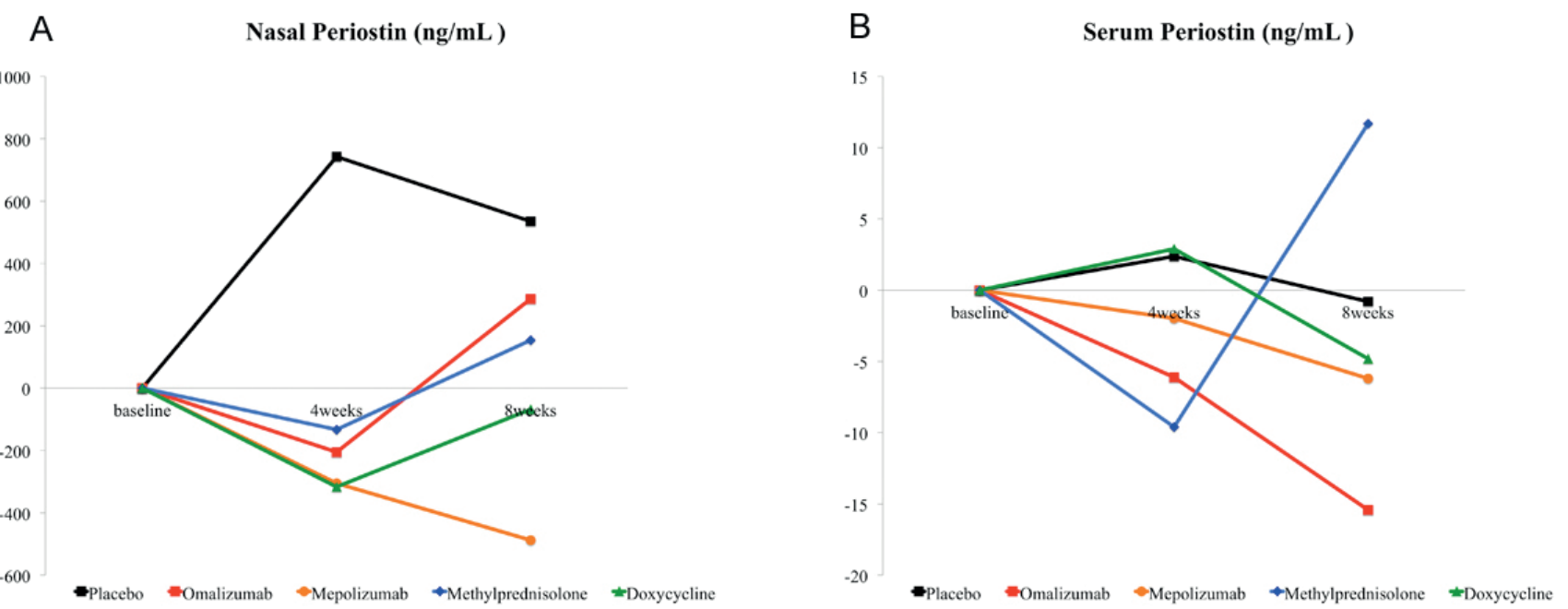

Figure 1. The effect of the different treatment options on nasal and serum periostin (mean change to baseline).

at 4 and 8 weeks after the start of the treatment.

Periostin was measured by means of an immunoassay (Genentech, South San Francisco) in preserved serum samples and nasal secretions, collected during the RCTs. The method of human specimen collection and processing has previously been described ${ }^{(22)}$. In short, serum tubes were collected and centrifuged at 3000 revolutions per minute (rpm) during 10 minutes. The serum is then pipetted into Eppendorf tubes and stored at $-20^{\circ} \mathrm{C}$. Nasal secretions were obtained by placing merocels (IVALON 4000 Plus $3,5 \times 0,9 \times 1,2 \mathrm{~cm}$ surgical products Fabco, New London, (T) in each nostril during 5 minutes. The merocels were weighted before and after the adsorption of secretions. Then $3 \mathrm{ml}$ of $0,9 \% \mathrm{NaCl}$ solution was added and after 2 hours the merocels are centrifuged at $1500 \mathrm{~g}$ during 15 minutes and stored at $-20^{\circ} \mathrm{C}$ until analysis.

Other key inflammatory markers were previously published and include nasal ECP, IL-5, sIL-5Ra, total IgE, MMP-9 and MPO; and serum ECP, sIL-5Ra, total IgE and peripheral blood eosinophil counts ${ }^{(14-16)}$. The clinical outcome was reproduced from previously published data and comprised the total polyp score (TPS) and the total symptom score. The size of nasal polyps was scored from 1 to 4 by means of endoscopy: 0 , no polyps; 1 , small polyps in the middle meatus not reaching below the inferior border of the middle concha; 2 , polyps reaching below the lower border of the middle turbinate; 3 , large polyps reaching the lower border of the inferior turbinate or polyps medial to the middle concha; and 4, large polyps causing complete obstruction of the inferior meatus ${ }^{(15,17)}$. The TPS is the sum of the right and left nostril scores. The total symptom score is the sum of individual symptom scores, namely anterior rhinorrhea, nasal obstruction, postnasal drip, and loss of sense of smell. All symptoms were scored as followed: 0 , no symptoms; 1 , mild symptoms; 2 , moderate symptoms; and 3 , severe symptoms.

\section{Ethics}

The ethics committee of the Ghent University Hospital approved all 3 studies and a written informed consent was obtained from all patients. The trials were undertaken in compliance with Good Clinical Practice guidelines and the ethics principles set out in the Declaration of Helsinki.

\section{Statistical analysis}

Baseline parameters were calculated and expressed in describing parameters and frequencies using the software program SPSS version 22. The Chi Square test was used to perform a post-hoc comparison of the prevalence of categorical baseline characteristics between groups. The non-parametric Kruskall Wallis test was used to compare baseline continuous variables and to verify that periostin levels are comparable between treatment groups. The non-parametric Spearman's Rank Correlation Coefficient $(\rho)$ describes correlations between baseline serum periostin and other parameters at baseline. Levels of periostin at 4 weeks and 8 weeks after the start of the treatment were compared to baseline levels in each group by means of the Wilcoxon test with Bonferroni correction.

The change in TPS was the primary end point in all three RCTs and statistics have been performed in the former studies ${ }^{(14,15,17)}$, therefore statistical analysis of clinical endpoints (TPS, TSS) was not repeated. We refer to the online repository for more information.

\section{Results}

\section{Patient characteristics at baseline}

The baseline characteristics are depicted in Table 1. Asthma ( $p=$ $0,001)$ and aspirin intolerance $(p=0,044)$ was significantly more present in the omalizumab treated patients whereas doxycycline treated patients were less frequently asthmatic. In this 


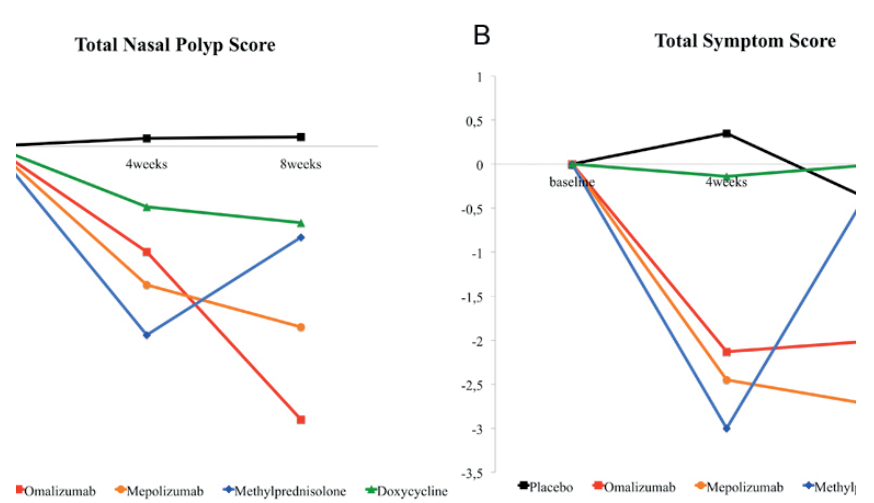

Figure 2. The effect of the different treatment options on total polyp scores and total symptom scores (mean change to baseline). These results are reproduced from the original trials.

context baseline nasal IL-5 ( $p=0.001)$, serum total $\lg E(p=0.022)$ and serum ECP ( $p=0.003$ ) significantly differed between groups. Baseline nasal periostin was significantly correlated with the asthma co-morbidity ( $p=0.011$ ) and serum levels significantly correlated with NSAID exacerbated respiratory disease at baseline. $(p=0.048)$. Periostin expression was higher in the nose as compared to serum $(p=0.052)$, but the difference in nasal and serum periostin between treatment groups was not significant (respectively $p=0.211$ and $p=0.563$ ).

\section{Change of nasal and serum periostin as compared to base- line}

Nasal periostin (Figure $1 \mathrm{~A}$ ) was significantly reduced by mepolizumab $(p=0.04)$ at 8 weeks after the start of the treatment. Nasal periostin levels were $401,5 \mathrm{ng} / \mathrm{mL}$ lower after 8 weeks of mepolizumab treatment (SEM 280,4; from $818,6 \mathrm{ng} / \mathrm{mL}$ to 417,1 $\mathrm{ng} / \mathrm{mL}$ ) (Table 1). Four weeks of doxycycline treatment reduced the mean nasal periostin by $206,1 \mathrm{ng} / \mathrm{mL}(\mathrm{p}=0.084)$.

Serum periostin (Figure 1B) was significantly reduced by methylprednisolone $(p=0.004)$ and by omalizumab $(p=0.004)$ respectively at 4 and 8 weeks after the start of the treatment. Four weeks of methylprednisolone treatment reduced the mean serum periostin with $11,1 \mathrm{ng} / \mathrm{mL}$ (SEM 2,6; from $73,9 \mathrm{ng} / \mathrm{mL}$ to $62,8 \mathrm{ng} / \mathrm{mL}$ ). Serum periostin levels were $15,5 \mathrm{ng} / \mathrm{mL}$ lower after 8 weeks of omalizumab treatment (SEM 5,0; from $90,0 \mathrm{ng} / \mathrm{mL}$ to $74,5 \mathrm{ng} / \mathrm{mL}$ ) (Table 1).

\section{Change of polyp and symptom scores}

The clinical parameters were published in the original studies and reproduced. The effects on TPS of the various treatment options were put in respect to each other. In the methylprednisolone treated group an early maximal decrease in polyp size was observed, but at week 8 the effect was extenuated. Administration of doxycycline reduced TPS in a gradual manner. Omalizumab and mepolizumab strongly improve polyp scores
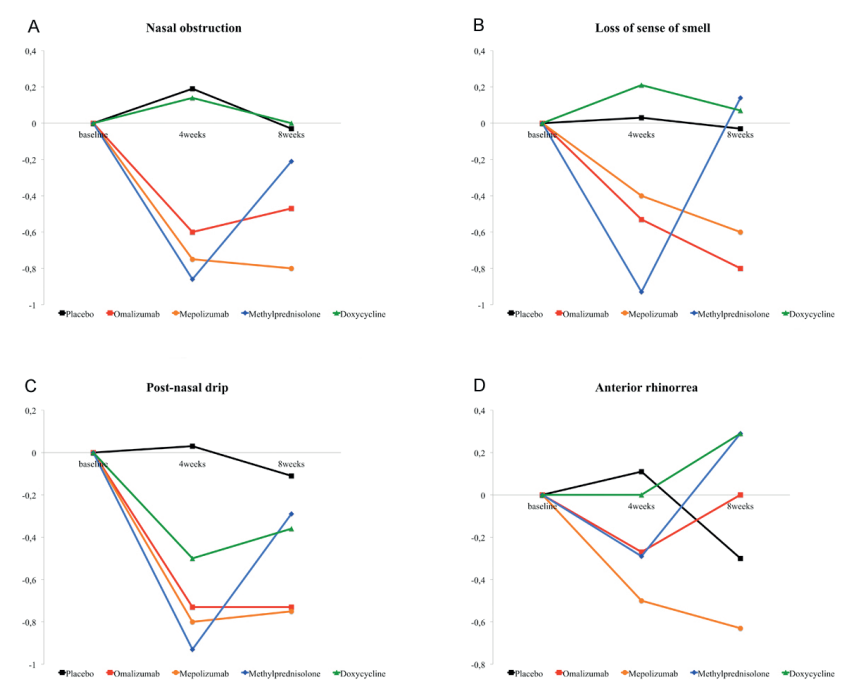

Figure E2. Influence of treatment on the total symptom score.

(Figure 2A).

All treatments markedly improve the total symptom score (Figure 2B) except for doxycycline, although it had an evident effect on post-nasal drip (Figure E2). Figure 2 illustrates the difference in effect of the different treatment options on the evolution of the total polyp score and total symptom score.

The mean change in periostin correlated significantly with the clinical improvement. The mean change in TPS correlated with the change in serum periostin ( $p=0.026$ at 8 weeks) and nasal periostin ( $p=0.015$ at 4 weeks). The mean change in TSS significantly correlated with the change in serum periostin (4 weeks $p=0.005 ; p=0.001$ at 8 weeks).

Overview of the effect of systemic treatments on periostin and on the inflammatory cascade

\section{Methylprednisolone}

In the methylprednisolone treated patients there was a significant reduction in serum periostin ( $p=0.004)$. There was also an early reduction of systemic and nasal eosinophilic markers at week 4. After cessation of oral steroid treatment, serum and nasal periostin expression mounted above baseline and blood eosinophil counts rebounded, as did all other eosinophilic biomarkers (Figure 3).

\section{- Doxycycline}

Nasal periostin was reduced after 4 weeks of treatment with doxycycline $(p=0.084)$, although this result did not reach significance. In accordance, other nasal inflammatory markers were inhibited and doxycycline most potently reduced local MMP-9 and local MPO (Figure 3).

\section{Omalizumab}

Omalizumab effectively blocked serum periostin (Figure 1) and interrupted the systemic eosinophilic cascade, whereas local markers remained unchanged (Figures 3-4). Nasal and serum 

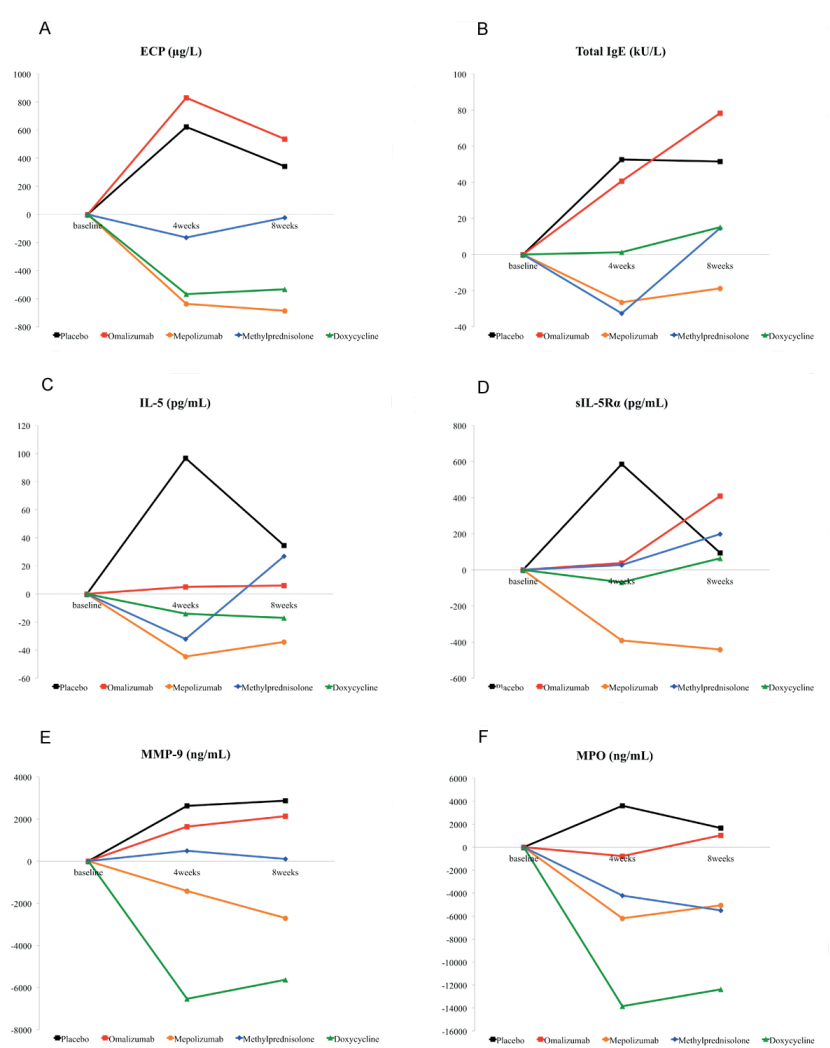

Figure 3. The effect of the different treatment options on local markers of inflammation, namely ECP, total IgE, IL-5, sIL-5Ra, MPO and MMP9 (mean change to baseline).

total IgE levels increased importantly during omalizumab treatment.

\section{- $\quad$ Mepolizumab}

Treatment with mepolizumab suppressed systemic and nasal periostin levels, the latter reached significance after 8 weeks of therapy $(p=0.044)$. Consistently, this treatment strongly reduced nasal and systemic key inflammatory markers (Figures 3-4) and depleted blood eosinophil counts, with mean values decreasing from $408,5 / \mu \mathrm{L}$ at baseline to $53,8 / \mu \mathrm{L}$ at 4 weeks and $47,5 / \mu \mathrm{L}$ at 8 weeks after the start of the treatment (Table 1).

\section{Placebo}

In accordance with periostin expression, local markers incremented in the placebo group (Figure 3), whereas serum markers generally remained unchanged (Figure 4).

\section{Discussion}

Each treatment distinctly interrupts the eosinophilic inflammatory cascade in CRSwNP, which is reflected by the local and systemic expression of periostin. Not only does the mechanism of action differ, but also the target site, which can be predominantly nasal, systemic or both.

- Methylprednisolone

Shortly after starting the treatment with methylprednisolone
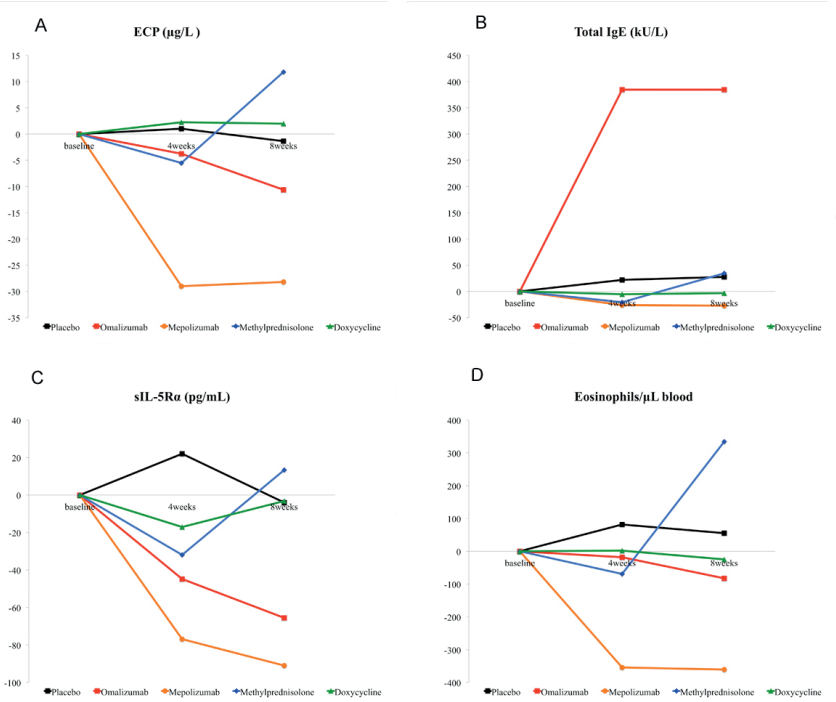

Figure 4. The effect of the different treatment options on systemic key markers of eosinophilic inflammation, namely ECP, total $\lg \mathrm{E}$, eosinophils and sIL-5Ra (mean change to baseline).

systemic periostin is significantly suppressed $(p=0.004)$. This beneficial effect is however followed by a rebound effect. In accordance, methylprednisolone potently but transiently reduces nasal and systemic TH2 inflammation and there is an overshoot after cessation of oral steroid treatment.

\section{Doxycycline}

The effect of doxycycline is mostly seen on nasal periostin, whereas serum periostin levels remain unchanged. Again this tendency is repeated in the other local and systemic inflammatory markers and therefore doxycycline appears to act at the target site. Next to its antimicrobial action it seems to protect the mucosa against inflammation by inhibiting local eosinophil degranulation and neutrophilic activity, and by reducing matrix degradation and thus edema ${ }^{(24)}$.

\section{- Omalizumab}

Omalizumab mainly interrupts the systemic eosinophilic inflammatory cascade. This is in line with the suppression of the systemic periostin expression ( $p=0.004)$. Of note, serum total $\lg \mathrm{E}$ levels augment due to the formation of omalizumab-lgE complexes with a more extended half-life. Therefore it is difficult to measure serum IgE correctly, as both free $\lg E$ and the complexes are detected. None of the treatment options decreases systemic IgE levels manifestly, as local and systemic markers of inflammation in CRSwNP are dissociated ${ }^{(25)}$ and serum IgE levels are not necessarily elevated in CRSwNP ${ }^{26,27)}$. Omalizumab potently improves clinical parameters, although the effect on the local inflammatory cascade equals the effect of placebo. Therefore the favorable clinical outcome in nasal polyp tissue seems to be mediated through a direct effect on IgE or its receptor. The suppressive effect on the systemic eosinophilic cascade is reflected 
by the effect on serum periostin, whereas the limited effect on nasal markers is mirrored by the temporary effect on nasal periostin.

\section{Mepolizumab}

The reduced expression of systemic and local periostin through elimination of the eosinophilopoietin IL-5 denotes an early interference in the inflammatory cascade, both locally and systemically.

\section{- Placebo}

Placebo does not affect systemic markers, although all markers of inflammation augment in nasal secretions in the placebo group, including periostin. We attribute this effect to the withdrawal of topical steroids, which have a suppressive effect on the local inflammation but not on systemic markers of inflammation.

Clinical implication: methylprednisolone, doxycycline, mepolizumab and omalizumab for treatment of CRSwNP

The change in serum periostin levels significantly correlates with clinical parameters; and it roughly parallels the trends described for the change in TPS and symptom scores for the different groups (see Figures 1B and 2A). Other key inflammatory markers change together with periostin.

Topical corticosteroids are the first choice drugs for the treatment of nasal polyps and this study underlines the importance of local treatment in this disease. We found a higher local periostin expression as compared to serum periostin; and nasal periostin increased throughout this study in the placebo group, although systemic markers remained unchanged. This finding is presumably due to the cessation of the intranasal steroids. Additional medical treatment can be indicated and has to be considered when topical treatment fails. Possibly the effect of a burst of oral steroids, as given in this study, is more extended in clinical practice where they are combined with nasal steroids. It would be interesting to follow-up periostin expression to establish treatment effect during such a combined treatment scheme. Anyhow, oral corticosteroids must be administered judiciously taking into account the side effects. Prescription is justifiable when associated with another treatment, or perioperatively to optimize surgical circumstances and to promote post-operative healing ${ }^{(14)}$.

Doxycycline is a low cost treatment that has an excellent bioavailability and has a long half-life. The latter promotes patient compliance as once-daily dosing is permitted. The interference with nasal periostin suggests that doxycycline is appropriate to prevent interrupt local inflammatory deterioration during exacerbations under the widespread treatment protocols with saline irrigations and intranasal steroids. The antimicrobial resistance associated with chronic low-dose tetracycline therapy is not clear, but repetitive regimens of doxycycline as an additive therapy could prevent recurrence of nasal polyps.
It would be interesting to explore the effect of methylprednisolone, which acts mainly through the systemic eosinophilic cascade, in combination with the locally active drug doxycycline. The complementary treatment effects might result in a better disease control. In case of remaining morbidity despite these non-specific treatments, surgery is the cornerstone. However, add-on therapy with monoclonal antibodies could be an alternative when early recurrence after surgery occurs despite conventional treatment or when patients are unfit for surgery. At present mepolizumab and omalizumab are not approved to treat CRSwNP. However, these treatments work very efficiently on nasal polyp size and symptoms related to nasal polyps in both asthmatic and non-asthmatics CRSwNP patients. Clinical features and molecular phenotyping, including measurement of periostin, could help to predict the local inflammation and identify the right patients for these expensive $\mathrm{TH}$ 2-targeted treatments. However, it is likely that not one, but a combination of systemic markers is necessary. Importantly, if omalizumab would become a treatment option for nasal polyps in the future, nasal IgE levels should be considered when calculating the treatment dose. Currently this is based on total serum IgE levels, and local $\lg \mathrm{E}$ is not considered. However, especially in non-asthmatics, inflammation in CRSwNP is primarily a local matter, as reflected by the higher nasal periostin expression as compared to serum levels.

\section{Limitations}

The dosage schedules specific to the type of drug treatments differ complicating the comparison of endpoints. For all treatments an anti-inflammatory effect is seen until month 2 in the initial studies, and therefore we compared the effect no longer than 8 weeks.

Secondly, separate randomized prospective trials were compared, resulting in different baseline characteristics. Only asthmatic patients were selected for the omalizumab trial, whereas asthma was less frequent in the doxycycline treated patients. This is reflected by the baseline nasal and serum periostin levels that were highest in omalizumab treated patients (resp. $1253 \mathrm{ng}$ / $\mathrm{mL}$ and $90 \mathrm{ng} / \mathrm{mL}$ ). Serum periostin at baseline was higher in all CRSwNP groups as compared to the values reported in literature for asthma patients ${ }^{(10,11)}$. However, we found that the frequency of asthma is proportional to periostin and other eosinophilic inflammatory markers. Furthermore, in contrast to CRSWNP, inflammation in asthma is reflected in the systemic circulation. This bias could influence the response to the tested treatments. Certain markers were measured in preserved sera or nasal secretions. Changes due to the different duration of storage and protease activity are not likely as the three studies were performed in approximately the same time period, and control samples were used to validate the test run.

Finally, topical steroids were withdrawn as these would influ- 
ence nasal inflammatory markers. However in clinical practices these treatments are added to standard-of-care intranasal corticosteroids. Therefore, the true impact for nasal polyp patients and the result might be even more obvious or long-term $(29,30)$. Recent trials more closely reflect the real life; for example treatment with anti-IL-4 (dupilumab) as add-on treatment has a proven beneficial effect on CRSWNP (31).

\section{Conclusion}

To conclude, our results show how each treatment has an individual effect on nasal and systemic inflammation. Methylprednisolone has an early and temporary effect on serum periostin and the systemic eosinophilic inflammation. Doxycycline predominantly affects nasal periostin, nasal inflammatory markers and markers of matrix degradation. Omalizumab interferes with serum periostin, the systemic eosinophilic cascade and acts directly through IgE. Finally, mepolizumab reduces both nasal and serum periostin expression and inhibits both eosinophilic and neutrophilic markers in the nasal mucosa and systemic circulation. The fluctuation in nasal and systemic periostin expression parallels the effect on other markers of inflammation and clinical response and therefore periostin is a promising marker for the anti-inflammatory response to a variety of treatments.

\section{Acknowledgements}

Els De Schryver receives funds from Special Research fund (BOF, B/11005/02). Philippe Gevaert receives a grant as a senior clinical investigator from the Flemish Scientific Research Board
(FWO). This work was also supported by grants to Claus Bachert from the Flemish Scientific Research Board (FWO, Nr. A12/5HB-KH3 and G.0436.04), and by the Interuniversity Attraction Poles Programs (IUAP) - Belgian state - Belgian Science Policy $\mathrm{P} 6 / 35$ and $\mathrm{P} 7 / 30$, and the Global Allergy and Asthma European Network (GA $\left.{ }^{2} L E N\right)$. Peter Hellings is the recipient of a post-doctoral mandate of the FWO Flanders. The opinions, results, and conclusions reported in this paper are those of the authors and are independent of the funding sources.

\section{Authorship contribution}

Philippe Gevaert, Claus Bachert and Thibaut Van Zele delivered the design of the study. Philippe Gevaert, Thibaut Van Zele, Peter Hellings and Lien Calus performed the original studies. Natalie De Ruyck and Gabriele Holtappels performed the laboratory analyses in Gent. Els De Schryver collected the data and performed the statistical analysis. Philippe Gevaert, Lara Derycke and Els De Schryver interpreted the data, drafted and finalized the manuscript. All authors critical revised the manuscript and approved the final version.

The opinions, results, and conclusions reported in this paper are those of the authors and are independent of the funding sources. The corresponding author had full access to all data in the study and had final responsibility for the decision to submit for publication.

\section{Conflict of interest}

No conflict of interest.

\section{References}

1. Hedman J, Kaprio J, Poussa T, Nieminen MM. Prevalence of asthma, aspirin intolerance, nasal polyposis and chronic obstructive pulmonary disease in a population-based study. Int J Epidemiol. 1999 Aug;28(4):717-22

2. Van Zele T, Holtappels G, Gevaert P, Bachert C. Differences in initial immunoprofiles between recurrent and nonrecurrent chronic rhinosinusitis with nasal polyps. Am J Rhinol Allergy. 2014 May-Jun;28(3):192-8

3. Fokkens W, Lund V, Mullol J. European position paper on rhinosinusitis and nasal polyps 2007. Rhinol Suppl.. 2007(20):1-136.

4. Gevaert P, Nouri-Aria KT, Wu H, Harper CE, Takhar P, Fear DJ, et al. Local receptor revision and class switching to IgE in chronic rhinosinusitis with nasal polyps. Allergy. 2013 Jan;68(1):55-63.

5. Gevaert P, Bachert C, Holtappels G, Novo $C P$, Van der Heyden J, Fransen L, et al. Enhanced soluble interleukin-5 receptor alpha expression in nasal polyposis. Allergy. 2003 May;58(5):371-9.

6. Lechapt-Zalcman E, Coste A, d'Ortho MP, Frisdal E, Harf A, Lafuma C, et al. Increased expression of matrix metalloproteinase- 9 in nasal polyps. J Pathol. 2001 Feb;193(2):23341.

7. Watelet JB, Bachert C, Claeys C, Van Cauwenberge P. Matrix metalloproteinases MMP-7, MMP-9 and their tissue inhibitor TIMP-1: expression in chronic sinusitis vs nasal polyposis. Allergy. 2004 Jan;59(1):5460.

8. Watelet JB, Claeys C, Van Cauwenberge P, Bachert C. Predictive and monitoring value of matrix metalloproteinase-9 for healing quality after sinus surgery. Wound Repair Regen. 2004 Jul-Aug;12(4):412-8.

9. Matsumoto H. Serum periostin: a novel biomarker for asthma management. Allergol Int. 2014 Jun;63(2):153-60.

10. Jia G, Erickson RW, Choy DF, Mosesova S, Wu LC, Solberg OD, et al. Periostin is a systemic biomarker of eosinophilic airway inflammation in asthmatic patients. J Allergy Clin Immunol. 2012 Sep;130(3):647-54 e10.

11. Hanania NA, Wenzel S, Rosen K, Hsieh HJ, Mosesova S, Choy DF, et al. Exploring the effects of omalizumab in allergic asthma: an analysis of biomarkers in the EXTRA study. Am J Respir Crit Care Med. 2013 Apr 15;187(8):804-11.

12. Rudmik $L$, Schlosser RJ, Smith $T L$, Soler ZM.
Impact of topical nasal steroid therapy on symptoms of nasal polyposis: a meta-analysis. Laryngoscope. 2012 Jul;122(7):1431-7.

13. Hissaria P, Smith W, Wormald PJ, Taylor J, Vadas $M$, Gillis D, et al. Short course of systemic corticosteroids in sinonasal polyposis: a double-blind, randomized, placebocontrolled trial with evaluation of outcome measures. J Allergy Clin Immunol. 2006 Jul;118(1):128-33.

14. Van Zele T, Gevaert P, Holtappels G, Beule A, Wormald PJ, Mayr S, et al. Oral steroids and doxycycline: two different approaches to treat nasal polyps. J Allergy Clin Immunol. 2010 May;125(5):1069-76 e4.

15. Gevaert P, Van Bruaene N, Cattaert T, Van Steen K, Van Zele T, Acke F, et al. Mepolizumab, a humanized anti-IL-5 mAb, as a treatment option for severe nasal polyposis. J Allergy Clin Immunol. 2011 Nov;128(5):989-95 e1-8.

16. Gevaert P, Calus L, Van Zele T, Blomme K, De Ruyck N, Bauters W, et al. Omalizumab is effective in allergic and nonallergic patients with nasal polyps and asthma. J Allergy Clin Immunol. 2013 Jan;131(1):110-6.e1.

17. Gevaert P, Calus L, Van Zele T, Blomme K, De Ruyck N, Bauters W, et al. Omalizumab is 
effective in allergic and nonallergic patients with nasal polyps and asthma. J Allergy Clin Immunol. 2013 Jan;131(1):110-6 e1.

18. Controlled Oral Steroid Intervention Decreases Serum Periostin Levels In Asthmatic Patients 2013.

19. Scheerens H, Arron JR, Choy DF, Mosesova S, Lal P, Matthews J. Lebrikizumab Treatment Reduces Serum Periostin Levels In Asthma Patients With Elevated Baseline Levels Of Periostin. J Respir Crit Care Med. 2012;185.

20. Hanania NA, Noonan M, Corren J, Korenblat $P$, Zheng Y, Fischer SK, et al. Lebrikizumab in moderate-to-severe asthma: pooled data from two randomised placebo-controlled studies. Thorax. 2015 Aug;70(8):748-56.

21. Corren J, Lemanske RF, Hanania NA Korenblat PE, Parsey MV, Arron JR, et al. Lebrikizumab treatment in adults with asthma. N Engl J Med. 2011 Sep 22;365(12):1088-98.

22. Watelet JB, Gevaert P, Holtappels G, Van Cauwenberge P, Bachert C. Collection of nasal secretions for immunological analysis. Eur Arch Otorhinolaryngol. 2004 May;261(5):242-6.

23. Shoenfeld $Y$, Gurewich $Y$, Gallant LA Pinkhas J. Prednisone-induced leukocytosis Influence of dosage, method and duration of administration on the degree of leukocytosis. Am J Med. 1981 Nov;71(5):773-8.

24. Rempe S, Hayden JM, Robbins RA, Hoyt JC. Tetracyclines and pulmonary inflammation. Endocr Metab Immune Disord Drug Targets. 2007 Dec;7(4):232-6

25. De Schryver E, Devuyst L, Derycke L, Dullaers M, Van Zele T, Bachert C, et al. Local immunoglobulin e in the nasal mucosa: clinical implications. Allergy Asthma Immunol Res. $2015 \mathrm{Jul} ; 7(4): 321-31$

26. Gevaert P, Holtappels G, Johansson SG, Cuvelier C, Cauwenberge P, Bachert C. Organization of secondary lymphoid tissue and local IgE formation to Staphylococcus aureus enterotoxins in nasal polyp tissue. Allergy. 2005 Jan;60(1):71-9.

27. Zhang N, Holtappels G, Gevaert P, Patou J, Dhaliwal B, Gould H, et al. Mucosal tissue polyclonal IgE is functional in response to allergen and SEB. Allergy. 2011 Jan;66(1):141-8.

28. Kim KS, Won HR, Park CY, Hong JH, Lee $\mathrm{JH}$, Lee $\mathrm{KE}$, et al. Analyzing serum eosinophil cationic protein in the clinical assessment of chronic rhinosinusitis. Am J Rhinol Allergy. 2013 May-Jun;27(3):e75-80.

29. de Borja Callejas F, Martinez-Anton A, Picado C, Alobid I, Pujols L, Valero A, et al. Corticosteroid treatment regulates mucosal remodeling in chronic rhinosinusitis with nasal polyps. Laryngoscope. 2015 May;125(5):E158-67.

30. van Camp C, Clement PA. Results of oral steroid treatment in nasal polyposis. Rhinology. 1994 Mar;32(1):5-9.

31. Bachert C, Mannent L, Naclerio RM, Mullol J, Ferguson BJ, Gevaert P, et al. Effect of Subcutaneous Dupilumab on Nasal Polyp Burden in Patients With Chronic Sinusitis and Nasal Polyposis: A Randomized Clinical Trial. JAMA. 2016 Feb 2;315(5):469-79.

Philippe Gevaert, MD, PhD Upper Airways Research Laboratory Department of Otorhinolaryngology Ghent University Hospital De Pintelaan 185

B-9000 Ghent

Belgium

Tel: +32-9332 2332

Fax: +32-0332 4993

E-mail: philippe.gevaert@UGent.be 